\title{
Neurofibroma of the peroneal nerve
}

\author{
Clayton Haldeman, MD, MHS, and Amgad Hanna, MD
}

University of Wisconsin, Department of Neurological Surgery, Madison, Wisconsin

Neurofibromas are benign tumors composed of different cell types from the peripheral nervous system. Neurofibromas infiltrate between nerve fascicles and do not have a discrete capsule. On MRI, they are T1 hypointense or isointense, T2 hyperintense, often with a "target sign," and contrast enhancing. The video shows gross-total resection of a peroneal nerve neurofibroma presenting as a painful mass in the popliteal fossa. Incisions across a skin crease can be either oblique or zigzag, but never perpendicular to it. It is also key to expose normal nerve proximal and distal to the tumor. The patient had a good functional outcome.

The video can be found here: https://youtu.be/G74Zoa1Y2JM.

KEYWORDS nerve sheath tumor; neurofibroma; peroneal nerve; video 